could provide the platform for commercially available graphene plasmonics detectors.

Published online: 23 October 2018

https://doi.org/10.1038/s41563-018-0207-1

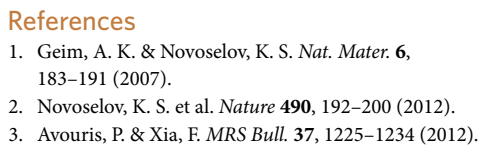

4. Guo, Q. et al. Nat. Mater. https://doi.org/10.1038/s41563-0180157-7 (2018).

5. Rogalski, A. Infrared Phys. Technol. 43, 187-210 (2002).

6. Rogalski, A., Martyniuk, P. \& Kopytko, M. Rep. Prog. Phys. 79, 046501 (2016).

7. Long, J. et al. Nat. Nanotech. 6, 630-634 (2011).

8. Fei, Z. et al. Nature 487, 82-85 (2012).

9. Chen, J. et al. Nature 487, 77-81 (2012).

10. Yan, H. et al. Nat. Nanotech. 7, 330-334 (2012).

\title{
Reading the atmosphere
}

ra ow much can you deduce about a material just by 'feeling' the space around it? It has been long known that electrically neutral matter creates an attractive van der Waals force in nearby matter, owing to correlated fluctuations in the materials' polarizabilities, which for a planar surface decays as the inverse cube of the distance. At distances of around several nanometres or so, the finite speed of the photons that transmit electromagnetic interactions alter ('retard') this interaction to change its distance dependence to $1 / d^{4}$, producing the Casimir force for conducting and dielectric surfaces. This force can equivalently be interpreted in terms of quantum fluctuations in the electromagnetic field of the intervening vacuum.

Descriptions of the Casimir force decades ago implied that it should show a materials dependence which, for certain combinations of materials, could even be made repulsive ${ }^{1}$. In other words, the force can be engineered. But one could equivalently say that the perturbation of the electromagnetic vacuum caused by a material may contain at least some information about the system causing it. In other words, one can learn about the material by probing its electromagnetic 'atmosphere'.

Qing-Dong Jiang and Frank Wilczek have now extended that idea by looking more broadly at how symmetry-breaking in the electronic structure of a material may manifest itself in what they call the 'quantum atmosphere', and how this might be used for materials diagnosis ${ }^{2}$. They consider, for example, topological insulators, in which the electronic structure violates parity (mirrorimage) and time symmetries. This symmetry-breaking introduces new contributions to the Casimir force near the material that could in principle be sensed from the perturbation it causes in the spectrum of a nearby probe atom or molecule, in this case by mimicking the effect of a magnetic field on the probe.

In this way a potential topological insulator might be diagnosed by a rather straightforward measurement of its quantum atmosphere. There's an echo here of the way surface plasmons are able to enhance the spectra of nearby probe molecules in surfaceenhanced Raman spectroscopy ${ }^{3}$, a technique for which the precise mechanism is still debated but which seems to be related to the translational symmetry-breaking of the surface itself.

The equations describing how the electromagnetic field interacts with a topological insulator turn out to be equivalent to those describing the interactions with hypothetical particles called axions, proposed in the 1970s (and later named by Wilczek) to explain a particular type of symmetry breaking in quantum chromodynamics, and now considered a candidate for dark matter.

Jiang and Wilczek say that other materials with exotic symmetrybreaking properties should also leave a characteristic imprint in their quantum atmosphere. This should be true, for example, for certain materials that contain chiral molecules, which

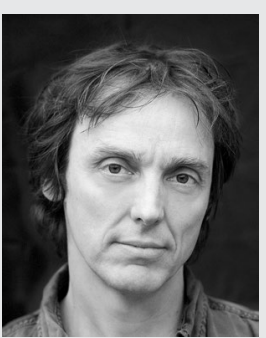

Philip Ball

may violate parity and cause polarized light to propagate at different speeds for left- and right-handed polarization (a so-called gyrotropic effect). Quite what the observable signature would be - and how large - would need to be calculated on a case-by-case basis.

A key question is whether these signatures would be strong enough to observe. Jiang and Wilczek argue that this should be feasible with existing technologies for topological insulators. They also speculate that probing quantum atmospheres might offer a way to identify new types of symmetry-breaking. The question then is how uniquely a particular (and perhaps faint) probe signature would specify the nature of the material causing it. Time (or is it parity?) will tell.

Published online: 23 October 2018 https://doi.org/10.1038/s41563-018-0213-3

References

1. Munday, J. N., Capasso, F. \& Parsegian, V. A. Nature 457, 170-173 (2009).

2. Jiang, Q.-D. \& Wilczek, F. Preprint http://www.arxiv.org/ abs/1809.01692 (2018).

3. Demirel, G. et al. J. Mater. Chem. C 6, 5314-5335 (2018). 